\title{
Conhecimento da população de Vassouras - RJ sobre Acidente Vascular Cerebral
}

\section{Knowledge of the population of Vassouras - RJ on Cerebral Vascular Accident}

Guilherme Thomaz Diniz Dumay ${ }^{\dagger}$, Juliana Ribeiro Milhomens Campelo ${ }^{\dagger *}$, Maria Paulina Viana Miquilino ${ }^{\dagger}, G_{a b r i e l a}$ Sudré Lacerda ${ }^{\dagger}$, Delfino Simões de Souza Neto ${ }^{\dagger}$,Carlos Eduardo Cardoso ${ }^{\ddagger}$, Ana Beatriz Calmon Nogueira da Gama Pereira $^{\S}$

Como citar esse artigo. Dumay, G.T.D Campelo, J.R.M; Miquilino, M.P.V; Lacerda, G.S; NETO, D.S.S; Cardoso, C.E; Pereira A.B.C.N.G. Conhecimento da população de Vassouras - RJ sobre Acidente Vascular Cerebral. Revista de Saúde. 2019 Jul./Dez; 10 (2): 02-06.

\section{Resumo}

O Acidente Vascular Cerebral (AVC) é uma doença muito prevalente e grave, apresentando alta morbimortalidade. Seus principais fatores de risco modificáveis são Hipertensão arterial sistêmica (HAS), tabagismo e diabetes mellitus e os não modificáveis são idade, gênero e raça. Os sinais clínicos têm rápido desenvolvimento, mas podem ser revertidos com o tratamento precoce, que só é obtido se a população for capaz de identificar corretamente seus sinais e sintomas.Inúmeras pesquisas identificam que o conhecimento geral é insuficiente. O presente estudo objetivou avaliar o conhecimento da população de Vassouras, RJ, sobre os fatores de risco relacionados à doença em questão para identificar se existe a necessidade de intervenções educativas sobre esta patologia.Tratou-se de um estudo observacional, transversal e qualiquantitativo cujos dados foram obtidos através de aplicação de questionário em uma Feira de Saúde em dois espaços públicos de grande circulação da cidade. Dos 119 participantes, 86,72\% responderam ter conhecimento sobre o que é AVC, enquanto 33,28\% desconhecem. Os fatores de risco com maior índice de acertos são HAS, fumo e níveis elevados de LDL e os de menor índice sãoAIDS, câncer e ronco. A maioria dos entrevistados que sabiam o conceito de AVC tinham maior escolaridade,porém há pacientes desconhecedores da patologia tanto no grupo de alta escolaridade quando de baixa, evidenciando a necessidade de conscientizar toda a população com campanhas que abordem tanto os principais fatores de risco quanto os sinais e sintomas para promover a rápida busca por tratamento.

Palavras-chave: Fatores de risco do AVE, Conhecimento leigo sobre AVE, Questionário de AVE

\begin{abstract}
Stroke is a very prevalent and serious disease, presenting high morbidity and mortality. Its main modifiable risk factors are systemic arterial hypertension (SAH), smoking and diabetes mellitus, and the non-modifiable ones are age, gender and race. Clinical signs have rapid development, but can be reversed with early treatment, which is only obtained if the population is able to correctly identify its signs and symptoms. Numerous researches identify that general knowledge is insufficient, so the present study aimed to evaluate the knowledge of the population of Vassouras, RJ on the risk factors related to the disease in question to identify if there is a need for educational interventions on this pathology. This is an observational, cross-sectional and qualiquantitative study whose data were obtained through the application of a questionnaire in a Health Fair in two public spaces of great circulation in the city. Of the 119 participants, $86.72 \%$ answered that they know what stroke is, while $33.28 \%$ do not know. The risk factors with the highest hit rate are SAH, smoking and high LDL, and the lowest risk factors are AIDS, cancer and snoring. Most of the interviewees who knew the concept of stroke had higher levels of education, but there are patients who are unaware of the pathology in both the high schooling and the low schooling group, evidencing the need to raise the awareness of the whole population with campaigns that address both the main risk factors and the signs and symptoms to promote the rapid search for treatment.
\end{abstract}

Keywords: Stroke risk factors, Lay knowledge about AVE, AVE Questionnaire

\section{Introdução}

O Acidente Vascular Cerebral (AVC) é uma emergência médica neurológica de grande prevalência e potencial de gravidade, apresentando altos índices de morbimortalidade ${ }^{1,2}$. De acordo com a etiologia, pode-se classificar o AVC em isquêmico, causado pela obstrução ao fluxo sanguíneo, ou hemorrágico, causado pelo extravasamento espontâneo de sangue em determinada área do cérebro. Nos dois casos, os sinais clínicos como alterações cognitivas e sensório-motoras tem rápido desenvolvimento, mas podem ser revertidos com o tratamento precoce através da rápida condução ao ambiente hospitalar, evitando, déficit permanente da função motora e cognitiva e queda da qualidade de vida que são situações de impacto tanto econômico quanto social, por causarem deficiências físicas, funcionais e emocionais ${ }^{1,2,3,4,5}$.

Os principais fatores de risco modificáveis do AVC 
são hipertensão arterial sistêmica (HAS), tabagismo e diabetes mellitus. Outros fatores como sedentarismo, obesidade e alcoolismo têm importância menor. Já os fatores de risco não modificáveis são idade, gênero e raça $a^{3,6}$.

A estratégia terapêutica mais eficaz para o AVC isquêmico é a administração de Alteplase IV, porém ela só pode ser realizada em até $4,5 \mathrm{~h}$ após o início dos sintomas, fato que evidencia a importância de reconhecer rapidamente o quadro ${ }^{7,8}$.

Acredita-se que uma maior conscientização da população sobre a gravidade do AVC e a identificação de seus sinais e sintomas podem aumentar as chances do paciente de receber o tratamento ideal e ter uma evolução satisfatória, já que esta patologia é uma das principais causas de incapacidade permanente e a principal causa de morte no Brasil ${ }^{1,2,7}$. Pesquisas em geral mostram que, apesar de campanhas de conscientização, existe alto índice de desconhecimento da população frente aos primeiros sinais e sintomas do $\mathrm{AVC}^{9}$. Diante de tal situação, o presente estudo objetivou avaliar o conhecimento da população do município de Vassouras, $\mathrm{RJ}$, sobre os fatores de risco relacionados à doença em questão, e analisar as características sócio demográficas dos entrevistados. A partir destes dados será possível identificar a necessidade de intervenções educativas para melhor conscientização da população sobre esta patologia.

\section{Metodologia}

\section{Desenho do estudo}

Tratou-se de um estudo observacional, transversal e quali-quantitativo, no qual um questionário foi aplicado a 119 voluntários residentes do município de Vassouras-RJ, durante a realização da Feira de Saúde da Semana do AVC em 2017.

\section{Instrumento de coleta de dados}

Utilizou-se um questionário elaborado pelos autores. A execução de um pré-teste foi realizada com 20 indivíduos para a adequação semântica das perguntas, resultando em um questionário de 30 perguntas divididas em três grupos: 1) dados sócio-econômicos demográficos (11 perguntas); 2) fatores de risco relacionados ao AVC (14 perguntas); 3) conhecimento sobre AVC (5 perguntas).

\section{Local de coleta de dados}

A aplicação do questionário foi realizada em uma Feira de Saúde promovida pela Liga Acadêmica de Neurologia e Neurocirurgia Dr. Hélcio Alvarenga em dois espaços públicos de grande circulação na cidade de Vassouras (Av. Expedicionário Oswaldo de Almeida Ramos e Rua Barão do Amparo).

Paralelamente à aplicação do questionário, este estudo incluiu a coleta de dados antropométricos (peso, altura, IMC e circunferência abdominal) e realizada a aferição da Pressão Arterial.

\section{Análise estatística}

A análise dos dados foi realizada por estatística descritiva e a construção dos gráficos e tabelas com o auxilio do software Excel 2010.

\section{Ética em pesquisa}

Esta pesquisa é parte do projeto intitulado "Rastreamento de doenças Neurológicas no município de Vassouras", enviado ao comitê de ética em pesquisa na Universidade de Vassouras, Vassouras, RJ (aprovado pelo CAAE 72807417.2.0000.5290). Todos os pacientes foram devidamente informados sobre o estudo em questão e autorizaram o uso das informações para publicação de resultados.

\section{Resultados}

Das 119 pessoas submetidas ao questionário, $42 \%$ eram do sexo feminino e $58 \%$ do sexo masculino. A média de idade dos participantes foi de 42,7 anos, sendo 18 anos a idade mínima e 84 a idade máxima. Deste total, $86,72 \%$ responderam que sabem o que é $\mathrm{AVC}$, enquanto $33,28 \%$ não sabem o que é AVC (Figura $1)$.

Para melhor compreensão dos resultados, a amostra foi dividida em grupos para avaliar o conhecimento sobre o que é AVC por faixa etária, de acordo com IBGE (Figura 2).

A frequência e porcentagem da escolaridade dos participantes estão representadas na Figura3, assim como a porcentagem por escolaridade do conhecimento sobre o que é AVC.

Os fatores de risco que tiveram maior porcentagem de acertos pela população estudada (Tabela 1) mostraram de $75 \%$ a $87 \%$ para LDL, Fumo e HAS; e os com menor porcentagem de acertos, de $5 \%$ a $22 \%$ para AIDS, Câncer e Ronco (Tabela 2).

\section{Discussão}

Devido à alta prevalência, aos altos índices de morbimortalidade e ao grande potencial incapacitante do AVC é fundamental que a população tenha conhecimento 


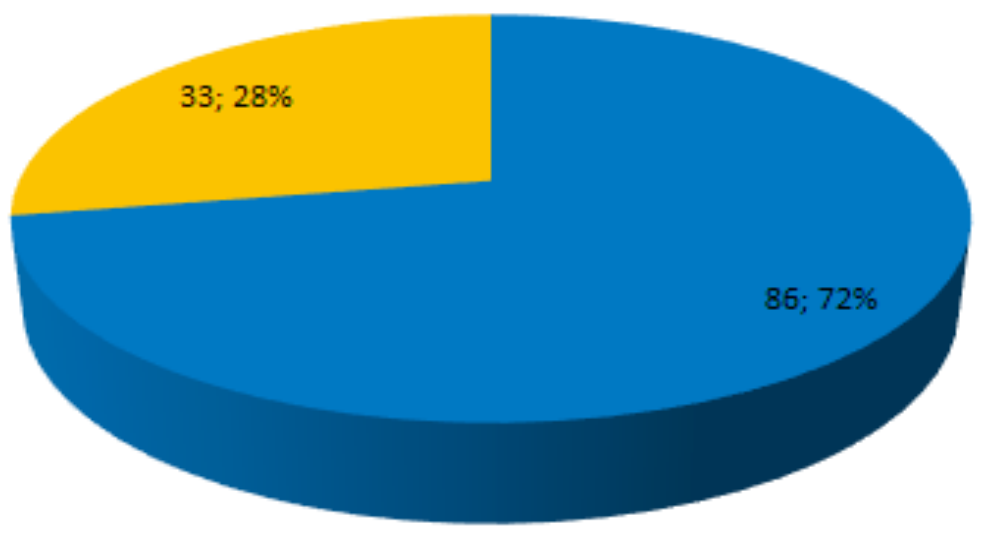

- Sabeo que éAVC Não sabe o que é AVC

Figura 1. Percentual da população total que sabe ou não o que é AVC

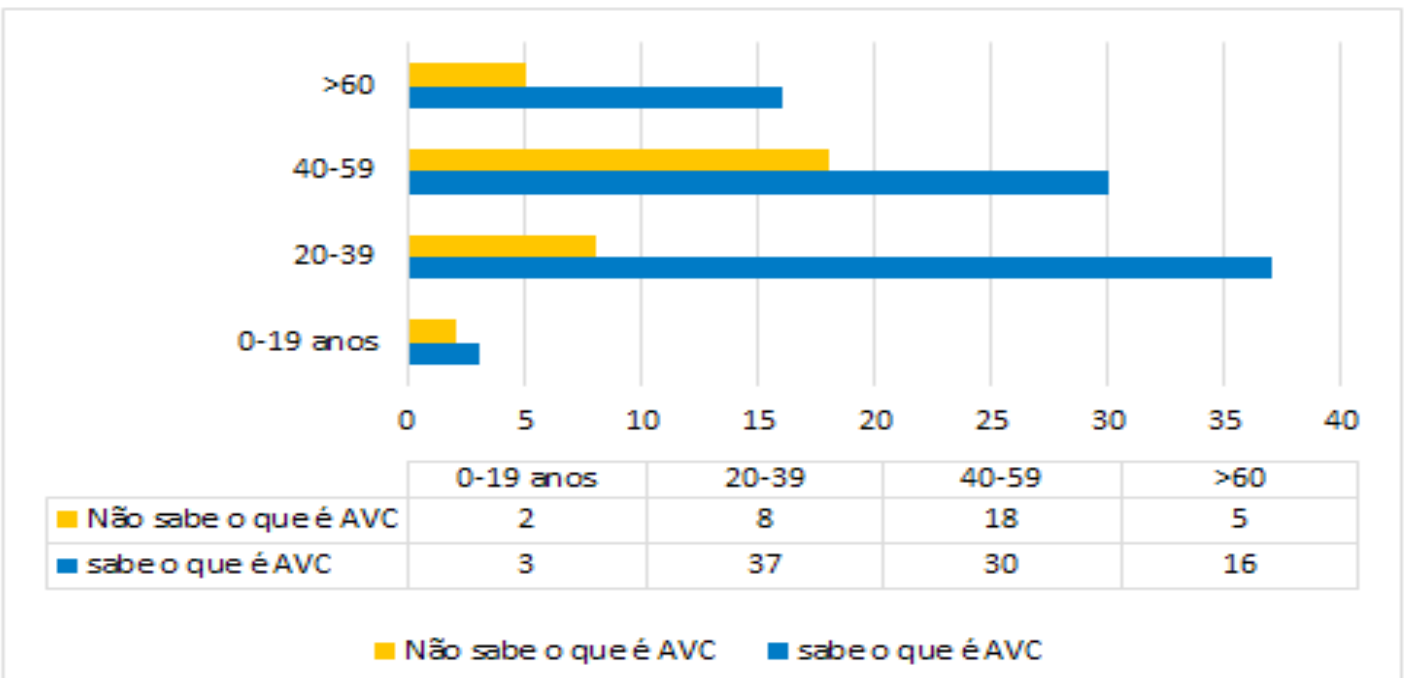

Figura 2. Valor absoluto de indivíduos que sabem ou não o que é AVC, separado por idade.

Tabela 1. Resultados com maior percentual de acertos, pela população em estudo, quanto aos fatores de riscos para AVC.

\begin{tabular}{c|c|c}
\hline Fator de Risco & Frequência de acertos & Porcentagem \\
\hline HAS & 104 & $87,4 \%$ \\
Fumo & 91 & $76,5 \%$ \\
LDL Alto & 90 & $75,6 \%$ \\
& & \\
\hline
\end{tabular}




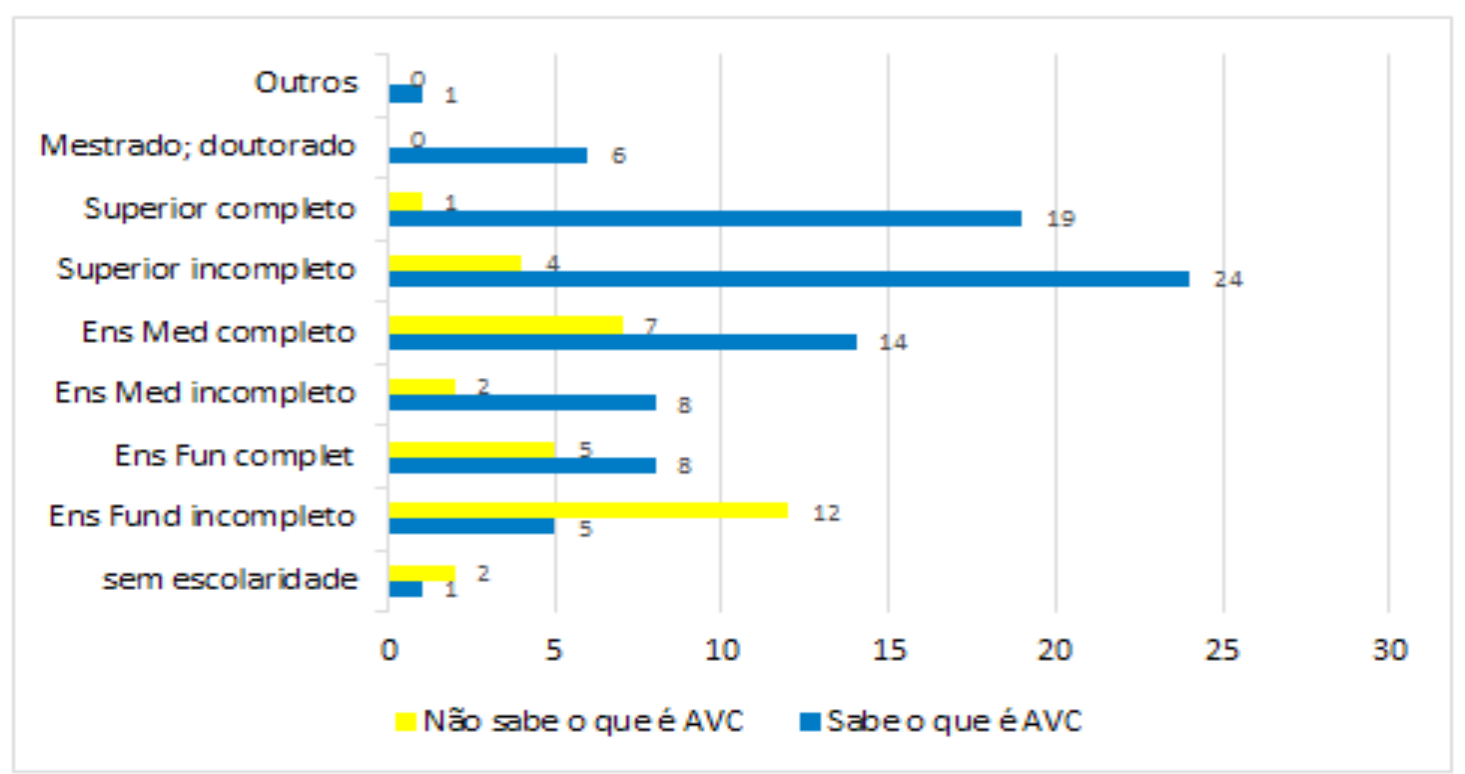

Figura 3. Valor absoluto de indivíduos que sabem ou não o que é AVC, separado por escolaridade.

Tabela 2. Resultados com menor percentual de acertos, pela população em estudo, quanto aos fatores de riscos para AVC

\begin{tabular}{c|c|c}
\hline Fator de Risco & Frequência de acertos & Porcentagem \\
\hline AIDS & 6 & $5 \%$ \\
Câncer & 15 & $12,6 \%$ \\
Ronco & 27 & $22,7 \%$ \\
& &
\end{tabular}

sobre seus fatores de risco, sinais e sintomas ${ }^{1}$. Este estudo demonstrou o nível de conhecimento da população de Vassouras, RJ, a fim de compará-lo com outros semelhantes para identificar as principais deficiências no conhecimento.

Apesar de ser uma patologia comum, a maioria dos entrevistados que sabiam o conceito de AVC tinham nível de escolaridade mais alto. Uma análise previa mostrou que apenas $28,72 \%$ dos moradores da zona urbana (ZU) de Vassouras e 17,85\% da zona rural (ZR) conheciam a correta nomenclatura da doença, enquanto que $49,40 \%$ na ZR e $39,89 \%$ na ZU utilizaram a palavra "derrame" para definir o distúrbio em questão ${ }^{1}$. Isto demonstrou que apesar de ser necessário utilizar os termos corretos nas campanhas de conscientização, é preciso fazer uma associação com a denominação utilizada pelo público leigo, visando facilitar a comunicação e alcançar o maior número possível de indivíduos.

A partir dos dados obtidos percebeu-se que existem cidadãos que desconhecem a patologia independentemente do nível de escolaridade, evidenciando a necessidade de fornecer informações, não só para as comunidades carentes, mas para toda a população. Tal fato já foi identificado entre pacientes que possuíam conhecimento prévio de AVC. Destes, $51,2 \%$ apresentaram consciência suficiente sobre fatores de risco e $54,9 \%$ sobre sinais e sintomas do $\mathrm{AVC}^{6}$.

No presente estudo, há relação diretamente proporcional entre conhecimento sobre AVC e nível de escolaridade. Já uma pesquisa realizada anteriormente mostrou maior conhecimento sobre os sintomas de AVC entre os moradores da ZR, previamente considerados mais desinformados por terem difícil acesso ao sistema de saúde, e ausência de diferença significativa quando 
comparou o conhecimento sobre fatores de risco para AVC entre moradores da ZR e ZU ${ }^{10}$.

Os fatores de risco mais destacados pelos entrevistados são HAS, alto LDL (lipoproteína de baixa densidade) e fumo. Semelhante fato foi relatado em diversos estudos que demonstraram mais de $80 \%$ de menção à hipercolesterolemia, doenças cardíacas e fumo. Adicionalmente, os termos "hipertensão, tabagismo, má alimentação, AVC prévio, estresse, sedentarismo e consumo de drogas" foram mencionados por cerca de $90 \%$ dos entrevistados ${ }^{1,11,12}$. O principal estilo de vida escolhido para prevenir AVC foi o que englobava boa alimentação, pratica de exercício físico e ausência de estress ${ }^{9}$. Esta situação pode ser considerada um avanço, pois reconhecer as causas que aumentam o risco para desenvolver uma patologia é um passo no caminho para preveni-la, principalmente quando se trata de fatores modificáveis.

Um quadro alarmante que se pode identificar é o desconhecimento sobre HIV e ronco como fatores de risco para AVC. Já foi encontrada uma incidência de AVC de 5,27/1000 pacientes em portadores de HIV e $3,75 / 1000$ pacientes em não portadores e mesmo após ajustes para idade, gênero e raça e controle para fatores de risco tradicionais a associação entre as duas patologias continuou significativa, sendo o HIV um preditor independente para patologia em questão ${ }^{13}$. Muitos estudos identificam a Síndrome de Apneia Obstrutiva do Sono (SAOS), cujo principal sintoma é o ronco, como um fator de risco isolado para $\mathrm{AVC}^{14}$. A partir disto, é notável a necessidade de falar principalmente sobre esses fatores menos conhecidos, para que a população possa tomar as medidas necessárias de prevenção.

Nesta pesquisa não há diferença significativa quando se relaciona idade com conhecimento prévio sobre AVC. Tal resultado é diferente do encontrado em um estudo prévio que demonstrou maior conhecimento entre os mais jovens ${ }^{10}$.

\section{Conclusão}

Diante do explicitado, é notável a necessidade de realizar campanhas de conscientização sobre AVC para toda a população, independente de classe social, local de residência ou idade, que abordem tanto os principais fatores de risco quanto os sinais e sintomas passíveis de reconhecimento para promover a rápida busca por tratamento e evitar sequelas. Tal conclusão baseia-se na presença de indivíduos que desconhecem a patologia tanto entre os de alta escolaridade quanto os de baixa.

Os resultados expostos mostraram que quanto maior o grau de escolaridade, maior o nível de conhecimento sobre AVC. Porém, há fatores de risco que são, em sua maioria, desconhecidos pela população, como HIV e ronco, ambos de risco independente para
AVC. Isto demonstra que o conhecimento geral sobre a doença é insuficiente, corroborando o encontrado pela maioria dos estudos descritos na literatura que fizeram o mesmo tipo de avaliação.

\section{Referências}

1. Silva IHB, Junqueira CM, Cortes PC, Cardoso RF, Marques CCS, Teixeira FCBD, et al. Conhecimento sobre o acidente vascular cerebral em VassourasRJ: Análise entre a população da zona urbana e zona rural. Neurobiologia. 2012;75(3):100-108

2. Pereira ABCN, Alvarenga H, Pereira Junior RS, Barbosa MTS. Prevalência de acidente vascular cerebral em idosos no Município de Vassouras, Rio de Janeiro, Brasil, através do rastreamento de dados do Programa Saúde da Família. Cad Saúde Pública. 2009;1929-1936.

3. Botelho TS, Machado Neto CD, Araújo FLC, Assis SC. Epidemiologia do acidente vascular cerebral no Brasil. Revista Temas em saúde. 2016;16(2):2447-2131.

4. Abe IM, Goulart AC, Santos Júnior WR, Lotufo PA, Benseñor IM. Validation of a stroke symptom questionnaire for epidemiological surveys. São Paulo Medical Journal. 2010;128(4):225-231.

5. Lima MJMR, Moreira TMM, Florêncio RS, Neto PB. Fatores associados ao conhecimento dos adultos jovens sobre histórico familiar de acidente vascular cerebral. Rev. Latino-Am. Enfermagem. 2016;24:1-9.

6. Fonseca LGA, Silva ALR, Pereira A. In: Anais do I Congresso Nacional de Envelhecimento Humano; 2016 nov. 23-25. Natal, Rio Grande do Norte. Rio Grande do Norte: Realize; 2016.

7. Coelho RS, Freitas WM, Campos GP, Teixeira RA. Stroke awareness among cardiovascular disease patients. Arq. Neuropsiquiatr. 2008;66(2A):209-212.

8. Powers WJ, et al. 2018 Guidelines for the Early Management of Patients with Acute Ischemic Stroke: A Guideline for Healthcare Professionals From the American Heart Association/American Stroke Association. Stroke. 2018; 49: 46-99.

9. Fernandes A, Magalhães CP, Mata MAP, Pimentel MH, Baptista MG. Avaliação do conhecimento referente à detecção precoce e prevenção do Acidente Vascular Cerebral. In: I Congresso de Cuidados Continuados: Dilemas Atuais e Desafios Futuros; 2012 set. 28-29. Bragança, Portugal. Bragança: Instituto Politécnico de Bragança, Escola Superior de Saúde; 2012 .

10. Ennen KA, Zerwic JJ. Stroke knowledge: how is it impacted by rural location, age, and gender?. Journal of Rural Nursing \& Health Care. 2010;10(1):9-21

11. Bule MJA, Sim-Sim MMS, Correia IMTB, Falé MJMM. Conhecimentos da população sobre acidente vascular cerebral - transeuntes da praça do giraldo em Évora. RevEnferm UFPE online. 2016;10(1):65-72.

12. Costa F, et al. Nível de conhecimento da população adulta sobre acidente vascular cerebral (AVC) em Pelotas - RS. J Bras Neurocirurg. 2008;19(1):3137

13. Chow FC, Regan S, Feske S, Meigs JB, Grinspoon SK, Triant VA Comparison of ischemic stroke incidence in HIV-infected and non-HIVinfected patients in a US Health Care System. Acquir Immune Defic Syndr. 2012;60(4):351-358

14. Silva GA, Sander HH, Eckeli AL, Fernandes RMF, Coelho EB, Nobre F. Conceitos básicos sobre síndrome da apneia obstrutiva do sono. Rev Bras Hipertens. 2009;16(3):150-157 